\title{
Vortex pseudo Schell-model source: A proposal.
}

\author{
Rosario Martínez-Herrero, \\ David Maluenda \\ and Gemma Piquero \\ Departamento de Óptica \\ Universidad Complutense de Madrid \\ Ciudad Universitaria 28040 Madrid, Spain \\ Email:r.m-h@fis.ucm.es
}

\author{
Juan Carlos González de Sande \\ ETSIS de Telecomunicación \\ Universidad Politécnica de Madrid \\ Campus Sur 28031 Madrid, Spain \\ Email: jcgsande@ics.upm.es
}

\begin{abstract}
In this work a physically realizable pseudo Schellmodel planar light source with optical vortex is proposed. The characteristics of irradiance and coherence of the beam radiated by this type of source are analysed at the source plane and upon free paraxial propagation.
\end{abstract}

\section{Introduction}

Schell-model sources has been found many applications in free-space optical communications, particle trapping etc and consequently have been receiving increasing attention in the literature, both in the scalar treatment [1]-[11] as in the vectorial case [12]-[15]. On the other hand, since their introduction, optical vortices are a subject of great interest [16]-[19] both from theoretical point of view as well as due its potential applications as particle manipulation. In this work a physically realizable planar light source with optical vortex and degree of coherence depending on the radius and on the phase difference is proposed. We will refer to it as a pseudo Shell-model source. At the source plane, it has the particularity of being completely coherent for any pair of points belonging to rings with center at the axis of the beam and partially coherent or incoherent for other pairs of points. Due to the phase that carries the optical vortex, this source presents a dark core at its center. The characteristics of irradiance and coherence of the beam radiated by this type of source are analysed at the source plane and upon free propagation.

Following this Introduction, in Section 2, the properties of the proposed CSD function are described, in Section 3, the free space propagation is analyzed, and finally, the main main results of this work are summarized in the Conclusion.

\section{Proposed cross spectral density function}

The most important constraint to ensure that a cross spectral density function (CDS) corresponds to a physically realizable source is to prove that such function is a non negative definite kernel. This may be a difficult task. However necessary and sufficient conditions has been stated in the literature [20]-[23]. In the present work we propose a source whose CSD at plane $z=0$ take the form

$$
\begin{aligned}
W\left(\mathbf{r}_{1}, \mathbf{r}_{2}, 0\right) & =\frac{2}{m ! \pi w_{0}^{2}}\left(\frac{\sqrt{2} r_{1}}{w_{0}}\right)^{m}\left(\frac{\sqrt{2} r_{2}}{w_{0}}\right)^{m} \\
& \times \exp \left[\operatorname{iim}\left(\varphi_{2}-\varphi_{1}\right)\right] \exp \left(-\frac{r_{1}^{2}+r_{2}^{2}}{w_{o}^{2}}\right) \\
& \times \frac{2 J_{1}\left(k b\left(r_{2}-r_{1}\right)\right)}{k b\left(r_{2}-r_{1}\right)}
\end{aligned}
$$

where $J_{1}(x)$ is the first order Bessel function; $\mathbf{r}_{j}=\left(r_{j}, \varphi_{j}\right)$ with $j=1,2$, are two arbitrary position vectors at the source plane, $m$ is a integer, $k$ is the wavenumber, $w_{0}$ is related to the source width, and $b$ is an adimensional parameter that controls the coherence area. The function is normalized in such a way that the total energy at the source plane is 1 .

By using the methods developed in [20]-[23], it can be proven that is a genuine CSD and therefore physically realizable source. The irradiance of the source can be obtained by evaluating the CSD at the same points [13], [24] and results

$$
I(\mathbf{r})=\frac{2}{\pi w_{0}^{2} m !} \exp \left(-\frac{2 r^{2}}{w_{o}^{2}}\right) .
$$

The degree of coherence [13], [24] of the proposed source is given by

$$
\gamma\left(\mathbf{r}_{1}, \mathbf{r}_{2}\right)=\exp \left[\operatorname{im}\left(\varphi_{2}-\varphi_{1}\right)\right] \frac{2 J_{1}\left(k b\left(r_{2}-r_{1}\right)\right)}{k b\left(r_{2}-r_{1}\right)}
$$

Taking into account Eqs. (1)-(3) we have the following properties of the source. It carries a topological charge $m$ and consequently presents a dark core at its center and then it presents a phase singularity. The absolute value of the degree of coherence only depends on the diference $r_{2}-r_{1}$ and therefore is equal to one, over rings for any choice of parameter $b$. More specifically, the degree of coherence as a function of $r_{2}-r_{1}$ presents a central lobe where the coherence is high and secondary lobes that are lower and lower. The first null of this function occurs for 


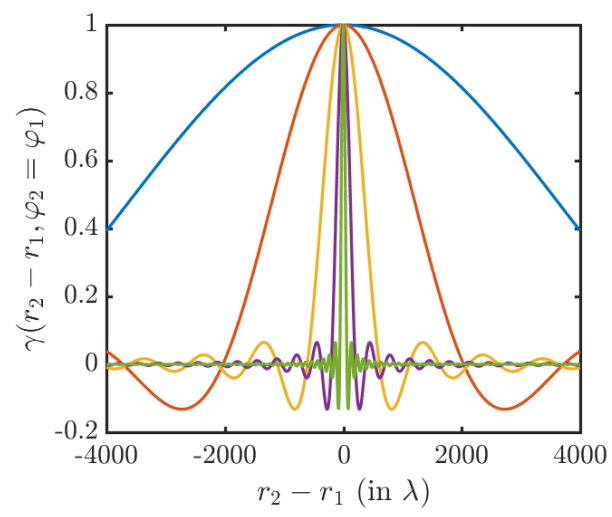

Figure 1. Degree of coherence for $b=0.1 \lambda / w_{0}$ (blue), $b=0.3 \lambda / w_{0}$, $b=\lambda / w_{0}, b=3 \lambda / w_{0}$, and $b=10 \lambda / w_{0}$ (green), for $w_{0}=1000 \lambda$.

$k b\left|r_{2}-r_{1}\right| \simeq 3.832$, which means a total bandwidth of the central lobe equal to $1.22 \lambda / b$. Fig. 1 shows the behaviour of the degree of coherence along radial direction $\left(\varphi_{2}=\varphi_{1}\right)$ for different values of parameter $b$.

On the other hand, the irradiance shows a donut shape, with a small (relative to $w_{0}$ ) dark core and the intensiyt being negligible at distances from the center greater than $2 w_{0}$ (see blue curve of Fig. 2). From Fig. 1 and the irradiance behavior, it can be deduced that for small enough parameter $b\left(b<<1.22 \lambda / w_{0}\right)$, the source is quasi coherent because the degree of coherence is nearly one for the whole area where the source has appreciable irradiance. On the contrary, when parameter $b$ is too large (well above $1.22 \lambda / w_{0}$ ), the source is nearly incoherent for arbitrary points, except for points that satisfy $r_{2}=r_{1}$. That is, when parameter $b$ is large enough, the source is completely coherent for any pair of points belonging to circles concentric to the center of the source and nearly incoherent for other pairs of points, even when this two points are very close if they are not on the same circle. For intermediate values of parameter $b$, the source is coherent for rings, presents a high degree of coherence for points in the vicinity of a given ring and is quite incoherent for points belonging to rings with considerably different radii. For any choice of $b$, the coherence is complete between points that satisfy $r_{2}=r_{1}=r$, although their distance can be as large as $2 r$.

\section{Free space propagation}

In order to obtain the CSD of these source at any plane, we assume paraxial propagation along $z$ axis, so the Fresnel diffraction integral can be used and the CSD is [24]

$$
\begin{aligned}
& W\left(\mathbf{R}_{1}, \mathbf{R}_{2}, z\right)=\frac{1}{\lambda^{2} z^{2}} \iint W\left(\mathbf{r}_{1}, \mathbf{r}_{2}, 0\right) \\
& \quad \times \exp \left[-\frac{\mathrm{i}}{2 z}\left(\left|\mathbf{R}_{1}-\mathbf{r}_{1}\right|^{2}+\left|\mathbf{R}_{2}-\mathbf{r}_{2}\right|^{2}\right)\right] d^{2} r_{1} d^{2} r_{2},
\end{aligned}
$$

where $\mathbf{R}_{\mathbf{j}}=\left(r_{j}, \theta_{j}\right)$ with $j=1,2$ are two arbitrary position vectors in $z$ plane.

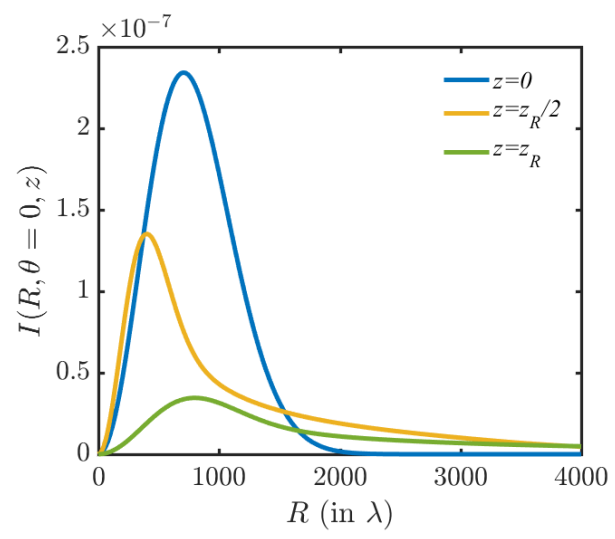

Figure 2. Intensity along radial direction at several distances from the source for a topological spiral charge $m=1, b=3 \lambda / w_{0}$, and $w_{0}=1000 \lambda$.

Substituting Eq. (1) into Eq. (4) and taking into account of Bessel functions definition, it can be found

$$
\begin{aligned}
W\left(\mathbf{R}_{1}, \mathbf{R}_{2}, z\right) & =\frac{8 \pi}{m ! \lambda^{2} z^{2} w_{0}^{2}} \exp \left[\frac{\mathrm{i}}{2 z}\left(R_{2}^{2}-R_{1}^{2}\right)\right] \\
& \times \exp \left[\mathrm{i} m\left(\theta_{2}-\theta_{1}\right)\right] \int_{0}^{\infty} \int_{0}^{\infty}\left(\frac{2 r_{1} r_{2}}{w_{0}^{2}}\right)^{m} \\
& \times \exp \left(-\frac{r_{1}^{2}+r_{2}^{2}}{w_{o}^{2}}\right) \frac{2 J_{1}\left(k b\left(r_{2}-r_{1}\right)\right)}{k b\left(r_{2}-r_{1}\right)} \\
& \times J_{m}\left(\frac{k}{z} R_{1} r_{1}\right) J_{m}\left(\frac{k}{z} R_{2} r_{2}\right) r_{1} r_{2} d r_{1} d r_{2} .
\end{aligned}
$$

Numerical evaluation of the integrals in Eq. (5) can be obtained. Propagation distance is expressed in terms of the Rayleigh distance $z_{R}=k w_{0}^{2} / 2$. For example, Fig. 2 shows that, after propagation, the decay rate of the irradiance for large $R$ is very low as compared with this decay rate at the source plane. Then, high values of irradiance extends in a wider area than at the source plane and remains large (relative to its maximum) for very large radius from the beam axis.

Regarding to the CSD, numerical simulation indicates that the area where the CSD takes high values increases with increasing values of the propagation distance. This means that the beam becomes more and more coherent with propagation distance.

\section{Conclusion}

In this work, a CSD representing a new vortex pseudo Schell-model source is proposed and analyzed. This source is completely coherent for any pair of points on a ring and is partially incoherent for points belonging to concentric circles with different radii. The distance in radial direction between two circles at which the degree of coherence reduces to zero is controlled by the parameter $b$ introduced in the definition of the source. This property, that is demonstrated at the source plane, seems to remain invariant in free space 
propagation. However the coherence area increases in free space propagation.

\section{Acknowledgments}

This work has been partially supported by Spanish Ministerio de Economía y Competitividad under project FIS2013-46475.

\section{References}

[1] A. T. Friberg and R. J. Sudol, "Propagation parameters of Gaussian Schell-model beams," Optics Communications, vol. 41, no. 6, pp. 383 $-387,1982$.

[2] F. Gori, G. Guattari, and C. Padovani, "Modal expansion for $J_{0^{-}}$ correlated Schell-model sources," Optics Communications, vol. 64, no. 4, pp. $311-316,1987$.

[3] A. T. Friberg and J. Turunen, "Imaging of Gaussian Schell-model sources," J. Opt. Soc. Am. A, vol. 5, pp. 713-720, May 1988.

[4] J. Serna, P. Mejías, and R. Martínez-Herrero, "Beam quality dependence on the coherence length of Gaussian Schell-model fields propagating through ABCD optical systems," Journal of Modern Optics, vol. 39, no. 3, pp. 625-635, 1992.

[5] H. Lajunen and T. Saastamoinen, "Propagation characteristics of partially coherent beams with spatially varying correlations," Opt. Lett., vol. 36, pp. 4104-4106, Oct 2011.

[6] S. Sahin and O. Korotkova, "Light sources generating far fields with tunable flat profiles," Opt. Lett., vol. 37, pp. 2970-2972, Jul 2012.

[7] Z. Mei and O. Korotkova, "Cosine-Gaussian Schell-model sources," Opt. Lett., vol. 38, pp. 2578-2580, Jul 2013.

[8] Y. Chen, F. Wang, C. Zhao, and Y. Cai, "Experimental demonstration of a Laguerre-Gaussian correlated Schell-model vortex beam," Opt. Express, vol. 22, pp. 5826-5838, Mar 2014.

[9] M. Santarsiero, G. Piquero, J. C. G. de Sande, and F. Gori, "Difference of cross-spectral densities," Opt. Lett., vol. 39, pp. 1713-1716, Apr 2014.

[10] F. Gori and M. Santarsiero, "Twisted Gaussian Schell-model beams as series of partially coherent modified Bessel-Gauss beams," Opt. Lett., vol. 40, pp. 1587-1590, Apr 2015.

[11] J. C. G. de Sande, M. Santarsiero, G. Piquero, and F. Gori, "The subtraction of mutually displaced Gaussian Schell-model beams," Journal of Optics, vol. 17, no. 12, p. 125613, 2015.

[12] F. Gori, M. Santarsiero, and R. Borghi, "Modal expansion for $J_{0-}$ correlated electromagnetic sources," Opt. Lett., vol. 33, pp. 18571859, Aug 2008.

[13] R. Martínez-Herrero, P. M. Mejías, and G. Piquero, Characterization of Partially Polarized Light Fields. Springer Series in Optical Science, Springer.

[14] F. Gori, V. Ramírez-Sánchez, M. Santarsiero, and T. Shirai, "On genuine cross-spectral density matrices," Journal of Optics A: Pure and Applied Optics, vol. 11, no. 8, p. 085706, 2009.

[15] Z. Mei and O. Korotkova, "Electromagnetic Schell-model sources generating far fields with stable and flexible concentric rings profiles," Opt. Express, vol. 24, pp. 5572-5583, Mar 2016.

[16] F. Gori, M. Santarsiero, R. Borghi, and S. Vicalvi, "Partially coherent sources with helicoidal modes," Journal of Modern Optics, vol. 45, no. 3, pp. 539-554, 1998.

[17] F. Gori, "Polarization basis for vortex beams," J. Opt. Soc. Am. A, vol. 18, pp. 1612-1617, Jul 2001.
[18] H. D. L. Pires, J. Woudenberg, and M. P. van Exter, "Measurements of spatial coherence of partially coherent light with and without orbital angular momentum," J. Opt. Soc. Am. A, vol. 27, pp. 2630-2637, Dec 2010.

[19] G. Gbur and T. D. Visser, "The structure of partially coherent fields," vol. 55 , pp. $285-311$.

[20] R. Martínez-Herrero, "Expansion of complex degree of coherence," Il Nuovo Cimento B, vol. 54, pp. 205-210, 1979.

[21] R. Martínez-Herrero and P. M. Mejías, "Radiometric definitions for partially coherent sources," J. Opt. Soc. Am. A, vol. 1, pp. 556-558, May 1984.

[22] F. Gori and M. Santarsiero, "Devising genuine spatial correlation functions," Opt. Lett., vol. 32, pp. 3531-3533, Dec 2007.

[23] R. Martínez-Herrero, P. M. Mejías, and F. Gori, "Genuine crossspectral densities and pseudo-modal expansions," Opt. Lett., vol. 34, pp. 1399-1401, May 2009.

[24] L. Mandel and E. Wolf, Optical Coherence and Quantum Optics. Cambridge University Press. 